\title{
Resolved star formation relations at high redshift from the IRAM PHIBSS program
}

\author{
Jonathan Freundlich ${ }^{1}$, Françoise Combes ${ }^{1,2}$, Linda Tacconi ${ }^{3}$, \\ Michael Cooper ${ }^{4}$, Reinhard Genzel ${ }^{3,5}$, Roberto Neri ${ }^{6}$ \\ and the PHIBSS consortium
}

\begin{abstract}
${ }^{1}$ LERMA, Observatoire de Paris, PSL Research University, CNRS, Sorbonne Universités, UPMC Univ. Paris 06, F-75014, Paris, France; ${ }^{2}$ Collège de France, PSL Research University, F-75005, Paris, France; ${ }^{3}$ Max-Planck-Institut für extraterrestrische Physik (MPE), Garching, Germany; ${ }^{4}$ Dept. of Physics \& Astronomy, Frederick Reines Hall, University of California, Irvine, CA, United States; ${ }^{5}$ University of California, Berkeley, CA, United States; ${ }^{6}$ IRAM,
\end{abstract} Grenoble, France

\begin{abstract}
Observed massive galaxies in the distant Universe form stars at much higher rates than today. High levels of star formation are sustained by a continuous supply of fresh gas and high molecular gas fractions. But after a peak around redshift $\mathrm{z}=2-3$, the star formation rate decreases by an order of magnitude. Is this evolution mostly driven by the available cold gas reservoir, or are the star formation processes qualitatively different near the star formation peak? The Kennicutt-Schmidt relation enables to characterize the star formation efficiency at low and high redshift, but resolved measurements at the scale of the star-forming regions themselves are still challenging at high redshift. Molecular gas observations carried out at the IRAM Plateau de Bure interferometer within the PHIBSS program (Tacconi, Combes et al.) permit us to study the star formation efficiency at sub-galactic scales around $\mathrm{z}=1.2$ and 1.5 for a limited sample of galaxies, and thus help characterize the star formation processes at this epoch. Our results lay in the continuation of the resolved low-redshift measurements, but further studies would be necessary to complement our sample and validate our conclusions.
\end{abstract}

Keywords. galaxies: evolution, galaxies: high-redshift, galaxies: structure, stars: formation

Ten billion years ago, between $\mathrm{z}=2-3$, observed galaxies formed their stars at rates up to 20 times higher than now (e.g., Madau \& Dickinson 2014). As stars are formed from cold molecular gas clouds, a high star formation rate (SFR) means a significant gas content. Galaxies near the star formation peak are indeed much more gas-rich and clumpy than their low-redshift counterparts (Tacconi et al.2010, 2013; Daddi et al.2010). Is the evolution of the cosmic SFR mostly driven by the declining cold gas reservoir, or are the star formation processes qualitatively different at high redshifts?

\section{Galaxy-averaged star formation from the IRAM PHIBSS program}

The IRAM Plateau de Bure High-z Blue Sequence CO(3-2) Survey (PHIBSS) studies the molecular gas properties of galaxies on the star-forming main sequence (MS) near the star formation peak. It notably aims at better understanding the winding down of star formation and the gas quenching processes. The Kennicutt-Schmidt (KS) relation between the gas and SFR densities enables to characterize the star formation efficiency, and is shown to be near linear at low redshift. The same trend is observed at $\mathrm{z}=1-3$ for the galaxies of the PHIBSS sample (Genzel et al.2010; Tacconi et al.2010, 2013). Nevertheless, the resulting global mean depletion time $\left(\mathrm{t}_{\mathrm{depl}}=\mathrm{M}_{\mathrm{gas}} / \mathrm{SFR}\right)$ of $0.7 \mathrm{Gyr}$ is slightly lower than at present time, which could imply a faster star formation duty cycle, more efficient star formation, and a need for gas replenishment at the peak of the star formation activity. 


\section{Resolved KS relations at sub-galactic scales}

The PHIBSS survey includes 8 high-resolution observations of the molecular gas, reaching an angular resolution of about 1" - which corresponds to $8 \mathrm{kpc}$ at $\mathrm{z}=1.2$. Resolved kinematics enables to separate smoothed ensembles of clumps due to their different velocities, and to obtain a resolved KS relation averaged at a scale below the spatial resolution for four $\mathrm{z}=1$ galaxies of the sample (Freundlich et al.2013). For one $\mathrm{z}=1.53$ massive star-forming galaxy (namely, EGS13011166), we also obtain a pixel by pixel KS relation (Genzel et al.2013). As shown in Fig. 1 , these results are compatible with a linear relation and a constant depletion time of about 1.9 Gyr, and fit well with the corresponding low-redshift observations. This seems to point towards similar star formation processes at high and low redshifts, but a bigger statistical sample would be necessary to obtain more meaningful mean depletion times for the substructures.

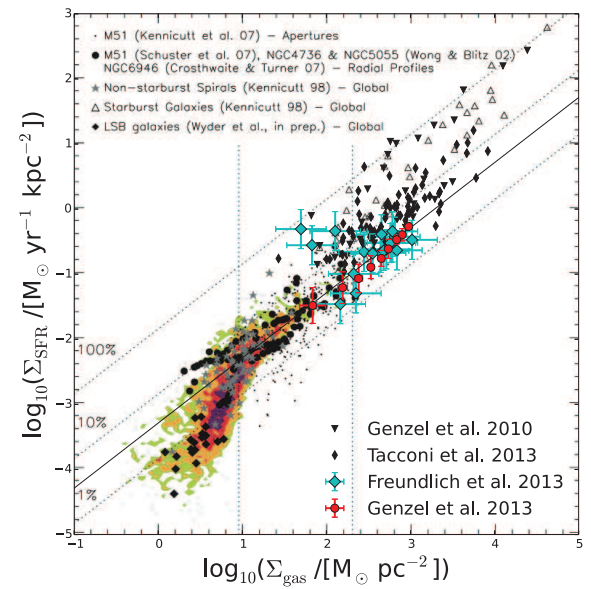

Figure 1. Spatially resolved molecular KS relation for ensembles of clumps of four galaxies from the PHIBSS sample (Freundlich et al.2013) and for binned groups of pixels in EGS13011166 (Genzel et al.2013), superimposed on the sub-galactic KS diagram obtained at low redshift by Bigiel et al.2008. The solid line corresponds to a constant depletion time $\mathrm{t}_{\mathrm{depl}}=1.9 \mathrm{Gyr}$, and black triangle and diamond points to galaxy-averaged measurements.

The ongoing IRAM PHIBSS2 Legacy Program (Combes, Garcia-Burillo, Neri, Tacconi, et al.) will more than triple the number of high-redshift MS galaxies with $\mathrm{CO}$ measurements, extend their redshift range, and include galaxies below the MS. Complementary ALMA programs will further contribute to extend the sample (Genzel et al.), probe higher $\mathrm{CO}$ transitions (Weiss et al.), and obtain high-resolution images and kinematics of the molecular gas in galaxies at intermediate redshifts (Freundlich et al.).

\section{References}

Bigiel, F., Leroy, A., Walter, F., et al. 2008, AJ, 136, 2846

Daddi, E., Bournaud, F., Walter, F. et al. 2010, ApJ, 713, 686

Freundlich, J., Combes, F., Tacconi, L. J., et al. 2013, A\& A, 553, 130

Genzel, R., Tacconi, L. J., Gracia-Carpio, J., et al. 2010, MNRAS, 407, 2091

Genzel, R., Tacconi, L. J., Kurk, J., et al. 2013, ApJ, 773, 68

Madau, P., \& Dickinson, M. 2014, ARA\&A, 52, 415

Tacconi, L. J., Genzel, R., Neri, R., et al. 2010, Nature, 463, 781

Tacconi, L. J., Neri, R., Genzel, R., et al. 2013, ApJ, 768, 74 